\title{
Verdrängung oder Sozialpolitik? Einfluss von Regulierungen auf die Wohneigentumsquote
}

\author{
Bisher stand die empirische Forschung zu Mietwohnungsmärkten nicht im Fokus \\ vergleichender Wohnungsforschung. Mit Blick auf die Debatte um steigende Mieten kann \\ vergleichende Forschung zum Mietwohnungsmarkt zum Erkenntnisgewinn beitragen. \\ Neue Daten aus 25 Industrieländern ermöglichen es nun, die Entwicklung der staatlichen \\ Regulierung von Mieten zu skizzieren. Dabei zeigt sich, dass stärkere Wohnraumlenkung \\ und intensivere Mietpreisregulierung zeitversetzt zu einem Anstieg der Wohneigentumsquote \\ beigetragen haben. Wohneigentum ist also mitunter eher indirekt durch budgetneutrale \\ Mietregulierungen als durch die teuren direkten Eigenheimsubventionen gefördert worden.
}

Seit 2010 ist in Deutschland ein kontinuierlicher Anstieg der Mietpreise zu beobachten. Damit ist die Frage nach der Bezahlbarkeit des Wohnens und eventuell notwendiger staatlicher Eingriffe auf die Agenda der öffentlichen Debatte gelangt: Was spricht für und was spricht gegen die Mietpreisbremse und andere preisregulatorische Maßnahmen? Eingriffe in die freie Preisbildung werden von Ökonomen oft kritisch gesehen. Sie stehen im Verdacht, den Neubau auszubremsen, Mietermobilität zu reduzieren und somit das Gefälle zwischen Bestandsund Neumietern zu verstärken. Die längerfristigen Auswirkungen einer solchen Regulierung, insbesondere auf die Größe des Mietwohnungsmarkts, sind hingegen kaum thematisiert worden. Zudem ist im Vergleich zur Forschung über Wohneigentum oder den sozialen Wohnungsbau auch die empirische Forschung zu Mietwohnungsmärkten, gerade im internationalen Vergleich, bisher eher vernachlässigt worden. ${ }^{1}$

Ein Grund dafür liegt in der starken Verbreitung von Wohneigentum. Selbst in Ländern mit einer niedrigen Wohneigentumsquote, zu denen die deutschsprachigen Länder zählen, hat sich die Zahl der Mieter langfristig betrachtet immer weiter reduziert. In fast allen anderen Ländern sind Mieterhaushalte längst zu einer Minderheit geworden. Nicht selten werden sie von der Politik als Noch-nichtEigentümer mit günstigeren Krediten bedacht oder als Nicht-mehr-Eigentümer mit stützenden Hilfsprogrammen

\footnotetext{
Der Beitrag beruht auf: K. Kholodilin, S. Kohl, Y. Prozorova, J. Licheron: Social policy or crowding-out? Tenant protection in comparative long-run perspective, Higher School of Economics Research Paper, Nr. WP BRP 202, St. Petersburg 2018.

1 Die meisten Studien konzentrieren sich auf die USA oder einige skandinavische Länder. Keine der uns bekannten Studien ist international vergleichend angelegt.
}

versorgt. Trotzdem sind sowohl im deutschsprachigen Raum als auch in Ländern mit einer hohen Eigentumsquote weiterhin in vielen Großstädten Mieterhaushalte weit verbreitet oder stellen eine nicht zu vernachlässigende Minderheit dar. Mit fortschreitender Urbanisierung und den wohneigentumsreduzierenden Effekten der Finanzkrise kann ihre Zahl sogar tendenziell zunehmen. Die Regulierung von Mietverhältnissen ist also immer noch ein wesentlicher Bestandteil von Wohnungspolitik.

\section{Mangelhafte Datenlage zu Mietregulierungen}

Ein weiterer Grund für die kaum vorhandene vergleichende Forschung zum Mietwohnungsmarkt liegt in einer mangelhaften Datenlage dieses stark vom nationalen Recht geprägten Bereichs. ${ }^{2}$ Die Datenlage ist nun

2 Vgl. auch S. Malpezzi, G. Ball: Measuring the Urban Policy Environment: An Exploratory Analysis Using Rent Controls, in: Habitat International, 17. Jg. (1993), H. 2, S. 39-52.

Dr. Konstantin Kholodilin ist wissenschaftlicher Mitarbeiter am Deutschen Institut für Wirtschaftsforschung (DIW) Berlin und Professor an der National Research University Higher School of Economics (NRU HSE) in St. Petersburg.

Dr. Sebastian Kohl ist wissenschaftlicher Mitarbeiter am Max-Planck-Institut für Gesellschaftsforschung in Köln. 
wesentlich verbessert worden: Dank neuer historischvergleichender Datensammlungen zur Entwicklung der Mietregulierung, des Wohneigentums und anderer Wohnungspreisindikatoren sind genauere Untersuchungen möglich. ${ }^{3}$ Die Kombination dieser Datensätze erlaubt es, der Frage nachzugehen, wie Mietregulierungen seit ihrer Einführung während des Ersten Weltkriegs in westlichen Ländern langfristig auf die Wohneigentumsquote gewirkt haben.

Die neuen Mietregulierungsdaten ${ }^{4}$ umfassen Regulierungsindizes zur Mietpreis-, Mieterschutz- und Wohnraumregulierung sowie zur Eigentumsquote in 25 Industrieländern seit dem Ersten Weltkrieg. Sie beruhen auf einer Sammlung aller relevanten historischen Mietgesetze dieser 25 Länder und einer inhaltlichen Kodierung von 18 binären Kategorien (z. B., ob ein realer oder nominaler Mietpreisstopp vorliegt, ob ein Kündigungsschutz für Mieter besteht oder ob es Abrissverbote gibt). Diese Kategorien werden zu Regulierungsindizes für die Bereiche Mietpreiskontrolle, Kündigungsschutz und Wohnraumlenkung aggregiert. ${ }^{5}$ Je höher der Index, der zwischen 0 und 1 schwankt, desto intensiver ist die Mietmarktregulierung.

\section{Geschichte der Mietregulierung}

Diese Daten ermöglichen es, die langjährige Entwicklung der Mietregulierung zu skizzieren. Am Beginn standen strikte Preiskontrollen als Teil des Konsumentenschutzes im Kriegssozialismus des Ersten Weltkriegs in den vom Krieg betroffenen Ländern Europas (vgl. Abbildung 1). Die Preisregulierung wurde in der Zwischenkriegszeit in abgeschwächter Form beibehalten, nahm im Zweiten Weltkrieg wieder die strenge Form an und verwandelte sich in der zweiten Jahrhunderthälfte in Kontinentaleuropa in Preiskontrollen der zweiten Generation, die inflationsangepasste Mietpreissteigerungen und die Setzung der Mieten bei Neuvermietungen auf dem Marktniveau zuließen. Die Kriegs- und Nachkriegsepisoden sind auch Phasen stark wohnraumlenkender Eingriffe in den Mietwohnungsmarkt gewesen,

3 K. Kholodilin, J. P. Weber, S. Sebastian: Die Mietwohnungsmarktregulierung der letzten 100 Jahre im internationalen Vergleich, in: DIW Wochenbericht, 45. Jg. (2018), H. 8, S. 453-464; S. Kohl: Homeownership, Renting and Society: Historical and Comparative Perspectives, London 2017; Ò. Jordà, M. Schularick, A. Taylor: The Great Mortgaging: Housing Finance, Crises and Business Cycles, in: Economic Policy, 85. Jg. (2016), H. 31, S. 107-152.

4 Der noch wachsende Datensatz des Rental Market Regulation Index ist bereits publiziert: https://www.remain-data.org/ (8.5.2019).

5 Siehe ausführlicher K. Kholodilin: Measuring Stick-Style Housing Policies: a Multi-Country Longitudinal Database of Governmental Regulation, DIW Discussion Papers, Nr. 1727, 2018; J. P. Weber: The Regulation of Private Tenancies - A Multi-Country Analysis, Dissertation, Regensburg 2017.
Abbildung 1

Intensität der Mietregulierungen
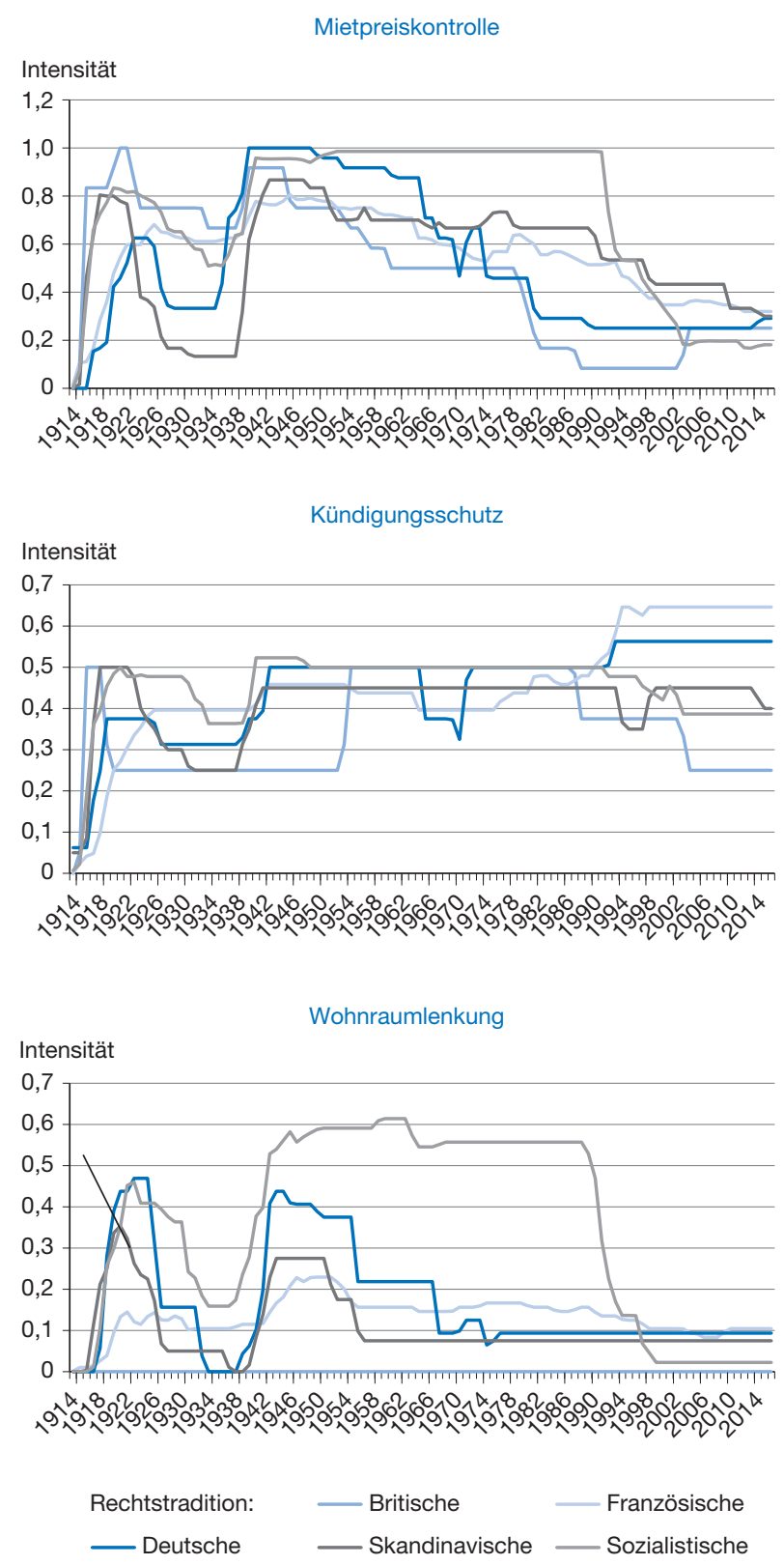

Quelle: eigene Darstellungen.

die weitgehend wieder zurückgefahren wurden, aber wie zuletzt der Milieuschutz oder Beschränkungen der kurzfristigen Vermietungen (wie Airbnb) - auch nicht völlig verschwanden, sondern eher in den letzten Jahren an Bedeutung gewannen. ${ }^{6}$ Der Kündigungsschutz bildete

6 Die zuletzt geäußerten Rufe nach Enteignungen privater Mietwohnungen können als weiterer Beleg dafür gesehen werden, dass Wohnraumlenkungsmaßnahmen noch als politische Optionen im Raum stehen. 
Abbildung 2

Wohneigentumsquoten nach Rechtstradition

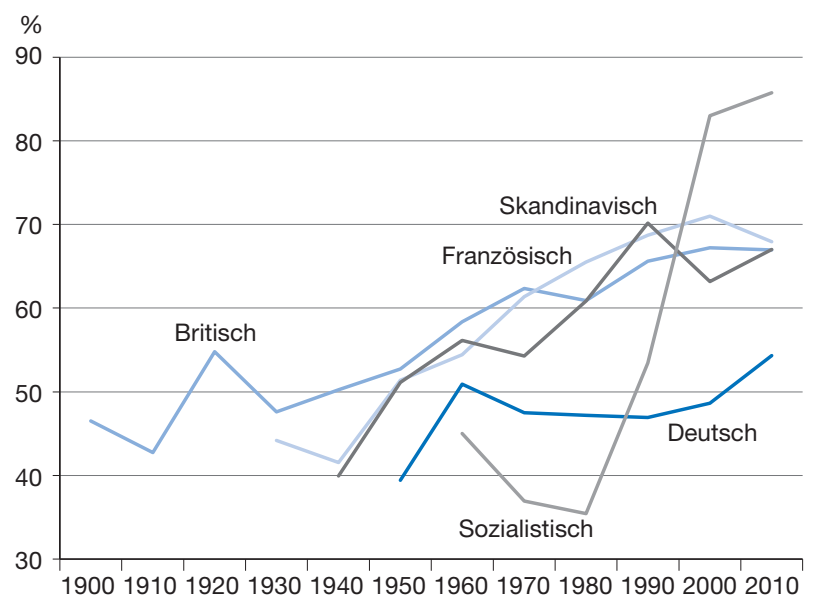

Quellen: CEPAL; Eurostat; UNSD; nationale statistische Ämter und eigene Darstellung.

sich ebenfalls im Zuge der ersten kriegssozialistischen Eingriffe heraus und wurde Bestandteil des neu entstehenden bürgerlichen Rechts für die Wohnungsmiete. Mietverhältnisse waren schon zuvor rechtlich reguliert worden, aber meist unspezifisch für Wohnungen als Mietobjekte, und enthielten höchstens Paragrafen gegen Wucher. Waren die Kriegsverordnungen noch Sonderrecht, überführten Nachkriegsregierungen diese dann in geregeltes bürgerliches Recht.

Diese knappe Skizze der Mietregulierungsgeschichte ist in erster Linie für zentral- und kontinentaleuropäische Länder wie Deutschland, Österreich oder die Niederlande charakteristisch, dabei unterscheiden sich verschiedene Länder und Ländergruppen erheblich voneinander. Schematisch kann man dieser Entwicklung die weitaus liberalere angelsächsische Entwicklung gegenüberstellen: Hier haben Mietregulierungen ihren Ausnahmecharakter meist nicht verloren und wurden mit dem Ende von Kriegen oder Inflationsschocks allmählich wieder abgebaut. In den USA etwa hat sich danach auch gar keine nationalstaatliche Mietpreispolitik entwickelt, daher können sich US-Städte sehr in ihrer Regulierungsgeschichte und -intensität unterscheiden. Auch hat sich in diesen Ländern der Mieterschutz nicht so stark entwickelt, sodass privates Mieten meist nur als eine vorübergehende Wohnform zweiter Klasse angesehen wird.

Dieser liberaleren Entwicklung steht die der sozialistischen Länder wie der DDR, den Ostblockstaaten oder den Sowjetrepubliken gegenüber, wo der private Miet- markt im Prinzip abgeschafft wurde. Nominal regulierte Preise bei völliger Unkündbarkeit des Mietverhältnisses und starken Eingriffen in den Wohnungsbestand perpetuierten in diesen Ländern die kriegssozialistischen Maßnahmen. Unter den westeuropäischen Ländern waren mietregulatorische Eingriffe in den süd- und nordeuropäischen Staaten etwas intensiver als im deutschen Rechtsraum.

Über diesen langen Zeitraum hinweg ist auch die Wohneigentumsquote in den westlichen Ländern fast kontinuierlich gewachsen. Abbildung 2 zeigt, dass die osteuropäischen Länder hingegen eine U-förmige Entwicklung durchlaufen haben, an derem Ende höhere Niveaus als im Westen standen. Im Westen selbst haben sich südeuropäische und einige nordeuropäische Länder zu Nationen mit einer hohen Wohneigentumsquote entwickelt, gefolgt von den früheren Wohneigentumsnationen angelsächsischen Ursprungs und deutschsprachigen Ländern als Schlusslichter dieser Entwicklung. Für die Entwicklung zu Wohneigentumsgesellschaften lässt sich eine Reihe von Gründen anführen: der allgemein gestiegene Wohlstand, das stetig fallende Realzinsniveau, aber auch eigenheimfördernde politische Maßnahmen in vielen Ländern.

\section{Regulierung kann Wohneigentumsquote erhöhen}

Diese traditionellen Erklärungen können in multivariaten Analysen durchaus bestätigt werden. So wurden Experten aus OECD-Ländern mit Blick auf eine Zeitspanne von mehreren Jahrzehnten befragt, wie ausgeprägt sie die Maßnahmen zugunsten des Eigentums einschätzen. Es stellte sich heraus, dass diese Indizes tatsächlich einen Teil des Anstiegs der Wohneigentumsquoten erklären können. ${ }^{7}$ Berücksichtigt man die Mietregulierungsindizes, wird diese erwartbare Erklärung noch weiter untermauert. Insbesondere lässt sich feststellen, dass stärkere Wohnraumlenkung und intensivere Mietpreisregulierung langfristig zu einem zeitversetzten Anstieg der Wohneigentumsquote geführt haben. ${ }^{8}$ Der Mietkündigungsschutz hingegen erweist sich als nicht signifikant. Auch ist der Einfluss der Mietpreisregulierung nicht linear-erreichen die Mietpreisregulierungen ein bestimmtes Niveau, führen noch intensivere Preisregulierungen nicht zu einem weiteren Anstieg der Wohneigentumsquote.

Die Ergebnisse legen eine Erklärung nahe, der bislang nur anekdotische Evidenz beigemessen wurde. Aller-

7 M. Atterhög: The Effect of Government Policies on Home Ownership Rates: An International Survey and Analysis, in: J. Doling, M. Elsinga (Hrsg.): In Home Ownership: Getting in, Getting from, Getting out Amsterdam 2006, S. 7-34.

8 Vgl. K. Kholodilin, J. P. Weber, S. Sebastian, a.a. O. 
dings wurde sie auch schon in einer historischen USStudie zu Wohneigentumssteigerungen im Zuge der US-Preiskontrolle während und nach dem Zweiten Weltkrieg angeführt: Starke Eingriffe in die Mietpreisgestaltung oder den Wohnungsbestand veranlassen Vermieter dazu, ihre Wohnungseinheiten an Mieter zu veräußern, die dies sowohl bei steigenden Einkommen als auch unter Wohnungsknappheit vermehrt nachfragen können bzw. müssen. ${ }^{9}$ Mit der Verbreitung alternativer Investitionsmöglichkeiten für Rentiers, wie etwa mobilere Finanzprodukte, war die Flucht der Vermieter aus dem Mietwohnungsmarkt auch eine realere Möglichkeit geworden. ${ }^{10}$

\section{Regulierung des Mietwohnungsmarktes als Sozialpolitik}

Mietregulierungen verschiedener Art sind oft mit guten Vorsätzen und tendenziell eher von linken Regierungen unterstützt worden, denn sie sind essenzieller Bestandteil einer Sozialpolitik für breite Bevölkerungsschichten im Bereich Wohnen. Berücksichtigt man, dass Wohnausgaben im 20. Jahrhundert Nahrungsausgaben als den größten Haushaltsbudgetposten abgelöst haben und dass auf dem Mietwohnungsmarkt häufig das "Schwabesche Gesetz" gilt,"11 wonach einkommensschwächere Haushalte höhere Mietkostenbudgetanteile

9 D. Fetter: The Home Front: Rent Control and the Rapid Wartime Increase in Home Ownership, NBER Working Paper, Nr. 19604, 2013.

10 C.-A. Michalet: Les placement des épargnants français de 1815 à nos jours, in: PUF, Paris 1968.

11 C. Dustmann, B. Fitzenberger, M. Zimmermann: Housing expenditures and income inequality, ZEW-Centre for European Economic Research Discussion Paper, Nr. 48, 2018. aufbringen müssen, ist Mietregulierungspolitik ein nicht zu unterschätzender Bereich staatlicher Sozialpolitik.

Allerdings zeigt sich aus historischer Perspektive auch, dass diese Eingriffe im Nebeneffekt zum graduellen Verschwinden des Regulierungsgegenstands Mietwohnungsmarkt in vielen Ländern beigetragen haben. Vermieter haben in vielen Ländern auf die wachsende Regulierung mit einer Umwandlung der bestehenden Mietwohnungen in Eigentumswohnungen reagiert und Neubauten von Geschosswohnungen fanden vermehrt in Form von Wohnungseigentum statt. Diese Prozesse führten zu einer langsamen Reduktion des Mietbestands zugunsten von Eigentumswohnungen.

Es waren nicht nur die Eigenheimträume konservativer und auch so mancher linker Parteien, deren Eigenheimförderung mit zu den heutigen Hauseigentumsgesellschaften geführt hat, ${ }^{12}$ sondern auch indirekt - so die These dieses Beitrags - die Entwicklung der Mietpreisregulierungen. Diese wurden vermutlich in den selteneren Fällen mit dem expliziten Ziel eingesetzt, Wohneigentum zu schaffen, obwohl im Spanien Francos oder auch unter norwegischen Sozialdemokraten die Verdrängung von Miet- zugunsten von Eigentumswohnungen mindestens in Kauf genommen wurde. In den häufigeren Fällen hingegen dürfte sich der Verdrängungseffekt vielmehr als eine langfristige Folge von eher kurzfristig gedachter Sozialpolitik zugunsten von Mietern eingestellt haben.

12 S. Kohl: The Political Economy of Homeownership: A Comparative Analysis of Homeownership Ideology through Party Manifestos, in: Socio-Economic Review, Online First, 2018, https://doi.org/10.1093/ ser/mwy030 (8.5.2019).

Title: Social Policy or Crowding-out? The Effect of Private-tenancy Regulation on Homeownership

Abstract: Using new data on the rent control, tenant protection and housing rationing as well as homeownership rates from 25 countries starting in the early 20th century up to the 2010s, the paper argues that stronger rent control and rationing of housing have contributed to the rise of homeownership. Originally passed as social policy to protect a considerable share of mostly urban residents, rent policies have rather crowded out the form of tenure they were meant to protect. They, thus, compete with the much more cost-intensive direct promotion of homeownership because they have made most countries homeownership-dominant over the past century.

JEL Classification: C23, 018, R38 